\title{
Effect of Acupressure on Cervical Ripening
}

\author{
Shahnaz Torkzahrani ${ }^{1}$; Khadighe Ghobadi ${ }^{2,3}$; Reza Heshmat ${ }^{4}$; Nezhat Shakeri ${ }^{5}$; Katayoun \\ Jalali Aria ${ }^{2,6^{*}}$ \\ ${ }^{1}$ Department of Midwifery, Faculty of Nursing and Midwifery, Shahid Beheshti University of Medical Sciences, Tehran, IR Iran \\ ${ }_{2}^{2}$ Counseling and Reproductive Health Research Center, Golestan University of Medical Sciences, Gorgan, IR Iran \\ ${ }_{3}^{3}$ Shahid Beheshti University of Medical Sciences, Tehran, IR Iran \\ ${ }^{4}$ Acupuncture Medicine Association, Tehran, IR Iran \\ 5 Acupuncture Medicine Association, Tehran, IR Iran \\ 6 Department of Midwifery, Faculty of Nursing and Midwifery, Golestan University of Medical Sciences, Gorgan, IR Iran \\ ${ }^{*}$ Corresponding Author: Katayoun Jalali Aria, Counseling and Reproductive Health Research Center, Golestan University of Medical Sciences, Gorgan, IR Iran. Tel: +989112733672, Fax: \\ +98-1732425171, E-mail: jalaliaria@yahoo.com
}

Received: March 30, 2015; Revised: May 22, 2015; Accepted: June 17, 2015

\begin{abstract}
Background: Cervical ripening is one of the main stages of initiation labor. Acupressure in Chinese medicine is considered as an invasive technique, which through reliving oxytocin ripens the cervix. Acupoint Sanyinjiao(SP6) was selected in this study because it is the acupoint selected in gynecology and it is easy for women to locate and apply pressure without medical assistance.

Objectives: The aim of this study was to determine the effect of acupressure on cervical ripening.

Patients and Methods: In this randomized clinical trial, 150 primigravida with term pregnancy who had referred to Deziani hospital in Gorgan were chosen and divided to three groups: in the first group acupressure was done by the researcher while in the second groups this was performed by the mother her self, and the third group served as a control and only received routine care. For both intervention groups the pressure was applied on Sp6 for about 20 minutes during one to five days. Elements were checked from cervical ripening at 48 and 96 hours after intervention and at the time of hospitalization. The tools for gathering information included demographic characteristics and midwifery history questionnaire, daily records and follow up forms. Content validity was used for validity of tools. Reliability of the observation check-list and physical examination was confirmed by inter-rater scores (inter observer), and daily records by test-re-test. Data was analyzed by analysis of variance (ANOVA), Kruskal-Wallis and Chi-squared tests ( $\mathrm{P} \leq 0.05)$.

Results: There was a significant difference between mothers' educations in the three groups. Most of the mothers (59.5\%) in the researcherperformed acupressure group had secondary education. Cervical ripening was significantly different between the three groups after 48 hours $(\mathrm{P} \leq 0.05)$,yet there was no significant difference after 96 hours and at the time of admission. Mean Bishop score was enhanced after 48 hours in the researcher-performed acupressure group $(\mathrm{P} \leq 0.021)$ and the self-performed acupressure group $(\mathrm{P} \leq 0.007)$ in comparison to the control group.

Conclusions: The results showed that acupressure is a safe technique and leads to cervical ripening. Thus, regarding the desired results that were achieved when mothers applied acupressure themselves, it could be suggested that it is beneficial for mothers to be trained to apply this method at home.
\end{abstract}

Keywords: Cervical Ripening; Acupressure; Labor; Acupuncture Points

\section{Background}

Cervical ripening is one of the main stages of initiation labor $(1,2)$. In fact the uterine cervix has to be firm through pregnancy and ripe enough before and during labor. Cervical ripening is a complex process and its mechanism is not well understood (3).

At the beginning of pregnancy, $50 \%$ of the cervix is made of collagen, $20 \%$ smooth muscle and the rest is elastin and glycosaminoglycans (GAGs), all embedded in a connective tissue ground substance (4).

Fibroblasts, the main cellular component of the cervix, produce collagen and GAGs. At the end of pregnancy, the collagen concentration of cervix decreases, water content increases, the GAGs composition changes, fibroblasts become active and local prostaglandin is produced. These fundamental, physiological and structural changes in the cervix cause cervical ripening. Cervical ripening needs an increase in prostaglandin production.

In this regard, prostaglandins are involved in changing the balance between various pro-inflammatory and antiinflammatory agents, which is one of the likely mechanisms of cervical ripening. By the process of cervical ripening, the cervix converts into a soft yielding structure, which has little resistance to the expulsive forces of the uterus and allows the fetus to pass through the birth canal during childbirth $(5,6)$. Favorable or ripe cervix is essential in order to stimulate the onset of labor. It allows full cervical dilation and effacement in the presence of uterine contractions $(7,8)$.

Iatrogenic cervical ripening is used commonly at the end of pregnancy. It is tantamount with procedures

Copyright (C) 2015, Iranian Red Crescent Medical Journal. This is an open-access article distributed under the terms of the Creative Commons Attribution-NonCommercial 4.0 International License (http://creativecommons.org/licenses/by-nc/4.0/) which permits copy and redistribute the material just in noncommercial usages, provided the original work is properly cited. 
used to artificially obtain cervical maturation or ripening and sometimes uterine contractions (2). The success of iatrogenic cervical ripening depends on the status of the cervix.

Bishop that was described by Edvard in 1964, is a system of pelvic scoring, which uses dilatation, effacement, station, cervical consistency and cervical position (Table 1) (8-10). This system is used for assessment of inducibility; a favorable Bishop score is defined as $\geq 6$ and in this situation the likelihood of successful induction is high. Scores of $\leq 6$ indicate reduced probability of successful induction and cervical ripening may be indicated (11).

The methods, which are utilized for cervical ripening, are divided to two groups: medical (prostaglandins and oxytocin) and non-medical (mechanical such as membrane stripping and complementary medication) $(5,12)$.

Among numerous cervical ripening methods, nonpharmacological approaches are more accepted by women. Acupuncture is one of the non-pharmacological methods, which due to enough scientific evidence supporting its effectiveness, has become increasingly used in labor wards (12).

As a subcategory of holistic medicine and a branch of acupuncture, acupressure is a method applied for induction of labor, which probably works based on oxytocin release induction mechanisms (13).

Acupuncture and acupressure mechanisms are based on the fact that there are 12 recognized main meridians and eight minor meridians, which are penetrable and comprehensible for the body and organs. These meridians are responsible for energy adjustment in the body and the source of diseases is by blockage of the energy stream. Acupuncture and acupressure are used as techniques for unblocking these occlusions. In general, 361 acupuncture points are located on these energy stream channels $(14,15)$.

Chinese medicine believes that energy channels or Q are stimulated by acupuncture or acupressure. This energy flows along 12 meridians, with special points along these meridians $(2,16)$. Acupressure is a technique that balances energy or Qi in the body (2). Meanwhile, Western medicine believes that stimulation of acupuncture or acupressure points may increase the release of prostaglandins and oxytocin $(2,16)$. Sanyinjiao (Sp6) is one of the important points, which is used in acupuncture, located on the spleen canal, and is applied in gynecology, extensively $(17,18)$.

Sanyinjiao is also stimulated for labor induction, transvers position of fetus and sedative labor pain (19). In a study conducted by Gaudet et al. (2008) in the Unites States of America, acupuncture decreased the average labor period in the intervention group compared to the control group (20).

However, the research done by Modlock et al. from Denmark indicated absence of any significant difference between the intervention and control groups in terms of the effect of acupuncture on induction of labor (12).

\section{Objectives}

Considering the controversies about the effectiveness of acupressure, this study was done with the aim of determining the effect of acupressure on cervical ripening.

\section{Patients and Methods}

The present study was performed in Gorgan (a northern city in Iran) of at the Golestan university of medical sciences of Iran, studying patients who had referred to the Dezyani medicine center from June 2011 to December 2012. This center was the only referral women's hospital in the Golestan province.

In this randomized clinical trial, 150 primigravida with term pregnancy who had referred to the Deziani hospital were chosen by the convenience sampling method. Regarding the following formula, the sample size for each group was 42 subjects yet considering patient loss during follow up, 50 subjects were allocated to each group.

(Confidence level $=95 \%$, test power $=80 \%$ )

$$
N_{1}=N_{2}=\frac{2\left(Z_{\frac{\alpha}{2}}+Z_{\beta}\right)^{2} \sigma^{2}}{\left(\mu_{2}-\mu_{1}\right)^{2}}
$$

Women were eligible for enrollment if they were nulliparous and had the following features, 39 to 41 weeks of gestational age confirmed by first-trimester ultrasound or normal last menstrual period, normal non-stress test, age of 18 - 35 years old, low risk pregnancy, Bishop score of $\leq 4$, single cephalic presentation, not taking herbal or chemical drugs 36 hours before and up to the end of the study, and not having sexual intercourse 24 hours before and up to the end of study. Exclusion criteria included taking chemical or herbal drugs, having sexual intercourse and being suspected of high-risk pregnancy.

The tools for gathering information were demographic characteristics and midwifery history questionnaire, daily record and follow up forms. These tools were prepared by the researchers and some were obtained from text books and articles. Content validity was used for validity of the tools. For this purpose the follow up forms and questionnaires were given to ten faculty members of Shahid Beheshti university of medical sciences and their point of views were considered.

Simultaneous validation method was applied to validate the accurate positioning of Sanyinjiao (Spleen 6) and the pressure insertion mechanism. In this regard, after passing a training course with an acupuncture specialist, the researcher cooperated with the specialist through applying pressure on the Sanyinjiao (Spleen 6) point on ten individuals and recorded their feelings including drowsiness, numbness, sore, hotness, coldness, pinching and pressing (kappa $=0.99$ ).

To study the validity of the designed observation and examination questionnaire, simulations of observation re- 
liability were used. In this regard, the researcher and two experienced midwives, (with 15 years of work experience) working in a maternity ward, performed separate vaginal examinations on 10 samples (kappa $=0.89$ ).

Subjects were randomly allocated to one of the three study arms: traditional Chinese medicine acupressure performed by the researcher, acupressure performed by the mother herself, and the control group whom only received routine care. Study arm assignment was determined by using a list of random numbers allocation (simple random allocation).

For the acupressure medicine group, the subjects were asked by the researcher to eat their breakfast and then refer to the hospital, between 9:00 am and 11:00 am; the interval between their breakfast and lunch meals should have been 45 to 60 minutes. Next, pressure was applied on the Spleen 6 spot for one to five sessions between 9:00 am and 11:00 am, which is the most effective period for pressure impact on Spleen 6 spot (14).

The pressure on this spot was applied for ten seconds to two minutes until half of the fingernails turned white, and then the spot was rested for the same period. This procedure was repeated for 20 minutes per day in one direction on the right foot and done every 24 hours, provided the subject didn't indicate laboring symptoms and childbirth. On the other hand, for the acupressure group, the mothers were trained about the acupressure procedure and received required information about the duration and time of the process, and submitted the daily record form to the research units. The spot of Spleen 6 was drawn on the daily record sheets of this group, so that the subjects could use it if necessary. The mothers were trained, and then practiced in the presence of the researcher. The third group was subjected only to routine healthcare services. The subjects were provided with the required trainings and then subjects of group 2 and 3 were asked to refer to the hospital 48 hours later, while subjects of group 1 visited the hospital on a daily basis. All three groups had a vaginal examination every 48 hours for determining their Bishop scores. Depending on initiation of childbirth symptoms, the number of acupressure sessions varied from one to five for each individual. Treatments of all three groups were followed by the researcher until the end of their hospitalization. The samples were asked to refer to the emergency unit of the hospital or call the researcher in the case of any labor pain, spotting or bleeding, rupture of membrane, reduced fetal movements or any other problems. The subjects were provided with the required trainings regarding fetal movements and labor initiation symptoms. Besides, they were taught about all research items such as herbal or chemical medicines and sex methods, and the obtained data were recorded in the related questionnaires. Furthermore, the phone number of the researcher was given to the subjects for them to contact around the clock in the case of having any question or problem. By initiation of labor symptoms or hospitalization for any reason, the patient was investigated by the researcher from the start of hospitalization to the end of labor and during the second and third trimester of labor. In this research, the sampling process was carried out for a six-month period, from $22^{\text {nd }}$ of June 2012 to $21^{\text {st }}$ of December 2012. At the end, among the 150 subjects, information of 133 mothers, including 42 subjects in the researcher-performed acupressure group, 41 subjects in the self-performed-acupressure group, and 50 subjects in the control group, were studied through statistical analysis and the remaining subjects were removed from the study due to reasons such as reluctance to pursue the study (four in group 1 and six in group 2), changes of childbirth hospital (three in group 1 and three in group 2 ), and lesion in pressure spot (one in group 1). However, no subject was removed from the routine care (control) group (Figure 1).

The SPSS16 software was used to analyze the data. Kolmogorov-Smirnov and Shapiro-Wilk tests were used to determine normal distribution of data. Data was analyzed by Analysis of Variance (ANOVA), Kruskal-Wallis, Mann-Whitney and Chi-squared tests using the SPSS16 software $(P \leq 0.05)$.

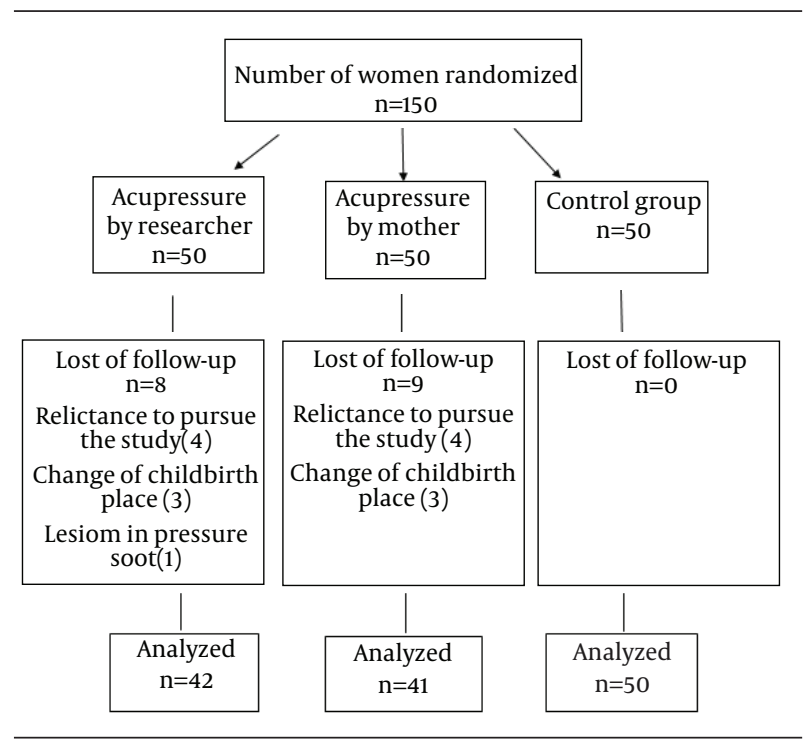

Figure 1. Flow Chart of Eligible Women

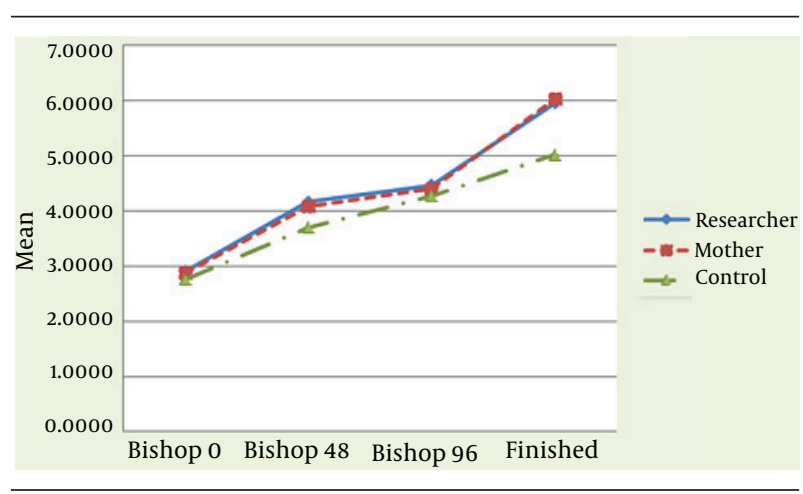

Figure 2. Trend of Bishop Score in Each Group 
To compare the means (age, gestational age, Bishop score before intervention) the Kruskal-Wallis test was used. Also for comparing Bishop Score means, KruskalWallis and ANOVA tests were used at the start of the study, after 48 and 96 hours, and at the time of hospitalization. Shapiro-Wilk test showed a normal distribution for the Bishop scores at the $49^{\text {th }}$ and $96^{\text {th }}$ hour and at the time of hospitalization.

Post-hoc was done to compare the groups. The protocol of this study was approved by the ethics and research committee of Shahid Beheshti university of medical sciences (No.400/2157, Date14/06/2011), and all subjects provided informed written consents to participate at the time of enrolment. The individuals were told that their information was kept private.

\section{Results}

Based on the findings of this research, these three groups were uniform and had no significant statistical differences in terms of mean age, career, ethnicity, gestational age, number of care services they attended, number of acupressure sessions in the two intervention groups, Bishop score at the start of the study, attendance of antenatal classes, and infant weight (Tables 2 and 3).

The average Bishop scores 48 hours after the beginning of the research were $4.88 \pm 1.83,5.12 \pm 1.92$, and 4.06 \pm 1.59 for the researcher-performed, self-performed, and control groups, respectively; implying no statistical difference among these three groups $(\mathrm{P}<0.012)$. Moreover, comparing researcher-performed and self-performed groups using the Mann-Whitney test after 48 hours of study indicated higher Bishop scores $(\mathrm{P}<0.021$ and $\mathrm{P}<$ 0.007 , respectively) as compared to the control group.

Although the Bishop score after 96 hours from the beginning of study indicated no significant difference $(\mathrm{P}=$ 0.95) among these three groups, the mean Bishop score in the acupressure groups was higher than that of the control group; the mean scores were $5.08 \pm 1.77,5.25 \pm 1.75$ and $5.04 \pm 1.96$ for researcher-performed, self-performed, and control groups (Figure 2).

Finally, the Bishop scores during hospitalization were $5.95 \pm 2.02,6.02 \pm 1.68$ and $5.02 \pm 2.03$, for researcher-performed, self-performed, and control groups, respectively; the ANOVA test indicated a significant statistical difference among these three groups $(\mathrm{P}<0.02)$ (Table 4$)$. The Dunnett test indicated differences in Bishop Scores of the researcher-performed acupressure group in comparison with the control group $(\mathrm{P}=0.028)$, and the self-performed acupressure group in comparison with the control group $(\mathrm{P}=0.014)$. The Friedman test indicated significant differences between the Bishop Scores of different groups (P $<0.000$ ) Bishop Score median and interquartile range (difference between the upper and lower quartiles) were showed in Table 5.

Table 1. Bishop Scoring System Used for Assessment of Inducibility

\begin{tabular}{|c|c|c|c|c|c|}
\hline Score & Dilatation, cm & Effacement, Percentage & Station $(-3$ to +2$)$ & Cervical Consistency & Cervical Position \\
\hline $\mathbf{0}$ & Closed & $0-30$ & -3 & Firm & Posterior \\
\hline $\mathbf{1}$ & $1-2$ & $40-50$ & -2 & Medium & Mid position \\
\hline 2 & $3-4$ & $60-70$ & -1 & Soft & Anterior \\
\hline 3 & $\geq 5$ & $\geq 80$ & $+1,+2$ & - & - \\
\hline
\end{tabular}

Table 2. Demographic Criteria of Pregnant Women Referred to Deziani Hospital a

\begin{tabular}{|c|c|c|c|c|c|}
\hline Variable & $\begin{array}{c}\text { Acupressure by } \\
\text { Researcher }(\mathrm{N}=42)\end{array}$ & $\begin{array}{c}\text { Acupressure by the } \\
\text { Mother }(N=41)\end{array}$ & $\begin{array}{c}\text { Routine Care } \\
(\text { Control })(\mathbf{N}=\mathbf{5 0})\end{array}$ & Test & PValue \\
\hline Ethnicity (Persian) & $33(78.6)$ & $26(63.4)$ & $37(74)$ & Chi-square & 0.28 \\
\hline Mother's education (High School) & $25(59.5)$ & $19(36.3)$ & $15(30)$ & Kruskal-Wallis & $<0.05$ \\
\hline Husband's education (High School) & $25(59.5)$ & $21(51.2)$ & $18(36)$ & Kruskal-Wallis & $<0.01$ \\
\hline Mother's occupation (Housekeeper) & $37(88.1)$ & $37(90.2)$ & $49(98)$ & Chi-square & 0.41 \\
\hline Participation in Antenatal classes & $4(9.5)$ & $1(2.4)$ & $3(6)$ & Chi-square & 0.398 \\
\hline Prenatal care & $42(100)$ & $41(100)$ & $50(100)$ & & 0.976 \\
\hline
\end{tabular}

a Data are presented as No. (\%).

Table 3. Means scores of the Studied Variables ${ }^{a}$

\begin{tabular}{|c|c|c|c|c|}
\hline Variables & $\begin{array}{c}\text { Acupressure by } \\
\text { Researcher } \mathrm{N}=42\end{array}$ & $\begin{array}{c}\text { Acupressure by the } \\
\text { Mother } N=41\end{array}$ & $\begin{array}{l}\text { Routine Care (Control) } \\
\qquad \mathbf{N}=\mathbf{5 0}\end{array}$ & PValue \\
\hline Mother's Age, y & $23.21 \pm 3.48$ & $22.54 \pm 3.47$ & $21.76 \pm 3.53$ & 0.17 \\
\hline Gestational Age, wk & $39.05 \pm 0.36$ & $39.63 \pm 0.42$ & $39.57 \pm 0.52$ & 0.506 \\
\hline Number of prenatal cares & $2.95 \pm 0.21$ & $2.95 \pm 0.21$ & $3 \pm 0.0$ & 0.976 \\
\hline Bishop Score & $2.90 \pm 0.589$ & $2.87 \pm 0.878$ & $2.76 \pm 0.74$ & 0.464 \\
\hline Infant Birth Weight, gr & $3358.21 \pm 371.25$ & $3332.07 \pm 409.54$ & $3195.20 \pm 403.17$ & 0.105 \\
\hline
\end{tabular}

${ }^{\mathrm{a}}$ Data are presented as mean $\pm \mathrm{SD}$. 
Torkzahrani S et al.

\begin{tabular}{|c|c|c|c|c|c|}
\hline Variable & $\begin{array}{c}\text { Acupressure by } \\
\text { Researcher }\end{array}$ & $\begin{array}{c}\text { Acupressure by } \\
\text { Mother }\end{array}$ & $\begin{array}{c}\text { Routine Care } \\
\text { (control) }\end{array}$ & Test & PValue \\
\hline 48 hours Bishop score & $4.88 \pm 1.83$ & $5.12 \pm 1.92$ & $4.06 \pm 1.59$ & Kruskal-Wallis & 0.012 \\
\hline Bishop $\geq 7$ & $7(16.7)$ & $13(31.7)$ & $6(12)$ & Chi-square & $<0.05$ \\
\hline 49-96 hours Bishop & $1.77 \pm 5.08$ & $1.75 \pm 5.21$ & $1.96 \pm 5.04$ & Anova & 0.95 \\
\hline Bishop $\geq 7$ & $7(16.7)$ & $5(12.2)$ & $8(16)$ & Chi-square & NS \\
\hline \multicolumn{6}{|l|}{ Hospitalization Time } \\
\hline Bishop & $2.02 \pm 5.95$ & $1.68 \pm 6.02$ & $2.03 \pm 5.02$ & Anova & $<0.02$ \\
\hline Bishop $\geq 7$ & $17(40.5)$ & $17(41.5)$ & $11(22)$ & Chi-square & 0.08 \\
\hline
\end{tabular}

${ }^{\mathrm{a}}$ Values are presented as Means \pm SD or No. (\%).

Table 5. Bishop Score Median and Inter Quartile Range(IQR)

\begin{tabular}{lccc}
\hline Group & $\begin{array}{c}\text { Before Intervention } \\
\text { Median (IQR) }\end{array}$ & $\begin{array}{c}\text { 48 Hours Later Me- } \\
\text { dian (IQR) }\end{array}$ & $\begin{array}{c}\text { 96 Hours Later Me- } \\
\text { dian (IQR) }\end{array}$ \\
\hline Researcher & $3(2)$ & $4(2)$ & $5(2)$ \\
Mother & $3(1.5)$ & $3.5(2)$ & $4.5(2)$ \\
Control & $3(1)$ & $3(1)$ & $4(2)$ \\
\hline
\end{tabular}

\section{Discussion}

In the present study, a significant difference in terms of Bishop score variation and cervical preparation (Bishop Score equal or greater than seven) was observed during the first 48 hours between the acupressure (particularly the one performed by mothers) and control groups. The findings of the present work are consistent with those of Azhari et al. (2009), who measured the effect of acupressure on labor initiation. The current research, indicated that the mean Bishop score within the first 48 hours of the study was significantly higher in the pressure group as compared to the touch group $(\mathrm{P}<0.008)$ and control groups $(P<0.001)(21)$. In another work conducted by Smith et al. (2008), the "effect of acupuncture on induction of labor" was investigated after two 45-minute sessions for two days; the difference between the Bishop scores of two real and simulated acupuncture groups were not statistically significant (13), which is inconsistent with the findings of the present work. Such difference can be attributed to the shorter intervention duration in the work of Smith et al. Besides, they did not compare their groups with a group who only received routine services.

In the present work, the mean Bishop Scores during the hospitalization indicated a significant difference among the three studied groups, which is in agreement with the results of Azhari et al. (21) Also, the results of Rabl et al. (2001) were in agreement with those of the present work. They aimed to determine the effect of acupuncture during cervical preparation and induction of labor at the second trimester of pregnancy. In their research, women who had referred to hospitals during the estimated date of confinement (EDC) were randomly divided to two acupuncture and control groups and then acupuncture was carried out for the intervention group at the Hegu (large intestine 4) and Sanyinjiao (spleen 6) spots. The results showed that cervical length in the acupuncture group was shorter than that of the control group, six and eight days after $\operatorname{EDC}(\mathrm{P}=0.04)(22)$.

Gribel et al. (2010) conducted a research titled "acupuncture for cervical preparation before induction of labor". Through this randomized clinical trial, 37 patients received acupuncture on different parts of their bodies (large intestine-4, stomach-36, large intestine-30, spleen-6, bladder-30, and bladder-23), while 35 were provided with misoprostol. Patients were hospitalized for 24 hours. Acupressure was done every seven hours up to a maximum of three sessions (6).

The results of this research showed no significant difference $(P=0.201)$ in the final Bishop scores of these groups, which is inconsistent with the findings of the present work. Such difference might be attributed to the procedure and participation criteria of their work. In their research, the acupuncture intervention period was shorter and there was no control group. Besides, the research units were nulliparous and multiparous and had Bishop scores below seven, which was different from the criteria of the present work.

Also, the results reported by Smith et al. (13) were different from those of ours. As previously mentioned, such difference can be explained by the shorter intervention period as well as selection of a greater number of acupuncture spots. Hence, for better efficacy of acupuncture, 
it is recommended to apply the procedure for ten days with two-day intervals (22).

Smith believed that as the most effective acupuncture spots among female organs are large intestine 4 and particularly spleen 6 , the efficacy of the acupuncturing process drops as the number of acupuncture spots increase. Another justification for this difference is that by increasing the number of spots, detection of simulated spots would be a difficult task as it results in intersecting the energy stream spots, which make comparing these two groups difficult (13).

In general, it can be stated that the present study has the following differences with the mentioned researches:

1. The research units were nulliparous females, with low variations of Bishop scores, as multiparity is among effective factors on Bishop scores. This point has not been considered by previous researches.

2. By selecting only one spot (spleen 6), which is the most effective spot in female organs, Bishop score variation in the first 48 hours of hospitalization was obtained, so that the acupressure process was more facilitated.

3. An intervention group of mothers was studied in the present work, whereas none of the mentioned previous researchers have studied this group.

Considering the fact that Bishop scores were higher in the self-performed group, on one hand, and only one effective spot had been selected in this work, on the other, training and applying acupuncture by mothers and intendants was facilitated. This innovation can be considered among the advantages of the present work. There was no placebo group in this study (pressure in null point).

Plenty of work has been conducted regarding the use of complementary and alternative medicines on females and midwifes and their effects have been investigated on gestation and labor. Considering the methodology of the present work, the nulliparous females were homogenous in terms of their age, career, age of gestation, Bishop score at the beginning of the study, number of prenatal cares, and participation in antenatal classes.

According to the findings of this research, acupuncture on the Spleen 6 spot could enhance cervical ripening (Bishop score equal or greater than seven) in the first 48 hours of study and during the hospitalization time. A comparison of these three groups indicated that acupressure performed the by mother her self could result in higher cervical ripening. Although cervical ripening did not show a significant statistical difference among the three groups after 96 hours from the start of the study, Bishop score increase and its growth percentage for the acupressure groups, particularly the self-performed group, was higher than that of the control group. Thus, it can be concluded that acupressure can be applied on the Spleen 6 spot for cervical ripening in pregnant women.

Finally considering the positive effect of acupuncture on the spleen 6 spot on cervical ripening, it can be concluded that this method can be applied easily, free and without side effects to improve maternal and infant's health. Furthermore, regarding the more desired results of the mentioned procedure in the self-performed acupressure group, this method could be taught to mothers to practice at home. In this way, it is possible to prevent problems concerning referring to the hospital and related costs.

\section{Acknowledgements}

This article was part of a student MS thesis. Particular thanks goes to Shahid Beheshti university of medical science's principals and Golestan university of medical sciences and the patients who cooperated in this project.

\section{Authors' Contributions}

Study concept and design: Shahnaz Torkzahrani and Khadige Ghobadi. Analysis and interpretation of data: Khadige Ghobadi and Shahnaz Torkzahrani. Drafting of the manuscript: Katayoun Jalali Aria and Khadige Ghobadi. Critical revision of the manuscript for important intellectual content: Shahnaz Torkzahrani. Statistical analysis: Khadigeh Ghobadi and Nezhat Shakeri. Acquisition of data: Khadighe Ghobadi. Administrative, technical, and material support: Khadighe Ghobadi and Reza Heshmat. Study supervision: Shahnaz Torkzahrani.

\section{Funding/Support}

This study protocol was approved by the ethics and research committee of Shahid Beheshti university of medical sciences (No.400/2157, Date14/06/2011), and all subjects provided informed written consent to participate on their date of enrollment. The individuals were told their information was kept private.

\section{References}

1. Blickstein I. Induction of labour. J Matern Fetal Neonatal Med. 2009;22 Suppl 2:31-7.

2. Tenore JL. Methods for cervical ripening and induction of labor. Am Fam Physician. 2003;67(10):2123-8.

3. Word RA, Li X H, Hnat M, Carrick K. Dynamics of cervical remodeling during pregnancy and parturition: mechanisms and current concepts. Semin Reprod Med. 2007;25(1):69-79.

4. Rayburn WF. Preinduction cervical ripening: basis and methods of current practice. Obstet Gynecol Surv. 2002;57(10):683-92.

5. Maul H, Mackay L, Garfield RE. Cervical ripening: biochemical, molecular, and clinical considerations. Clin Obstet Gynecol. 2006;49(3):551-63.

6. Gribel GP, Coca-Velarde LG, Moreira de Sa RA. Electroacupuncture for cervical ripening prior to labor induction: a randomized clinical trial. Arch Gynecol Obstet. 2011;283(6):1233-8.

7. Drzewiecki G, Tozzi C, Yu SY, Leppert PC. A Dual Mechanism of Biomechanical Change in Rat Cervix in Gestation and Postpartum: Applied Vascular Mechanics. Cardiovascular Engin. 2005;5(4):18793.

8. Laughon SK, Zhang J, Troendle J, Sun L, Reddy UM. Using a simplified Bishop score to predict vaginal delivery. Obstet Gynecol. 2011;117(4):805-11.

9. Edwards RK, Richards DS. Preinduction cervical assessment. Clin Obstet Gynecol. 2000;43(3):440-6.

10. Pennell CE, Henderson JJ, O'Neill MJ, McCleery S, Doherty DA, Dickinson JE. Induction of Labor in Nulliparous Women With an 


\section{Torkzahrani S et al.}

Unfavorable Cervix: A Randomized Controlled Trial Comparing Double and Single Balloon Catheters and PGE2 Gel. Obstet Gynecol Surv. 2010;65(2):78-80.

11. Norwitz ER, Schorge JO. Obstetrics and Gynecology at a Glance.New York City: John Wiley \& Sons; 2013.

12. Modlock J, Nielsen BB, Uldbjerg N. Acupuncture for the induction of labour: a double-blind randomised controlled study. BJOG. 2010;117(10):1255-61.

13. Smith CA, Crowther CA, Collins CT, Coyle ME. Acupuncture to induce labor: a randomized controlled trial. Obstet Gynecol. 2008;112(5):1067-74.

14. Stux G, Berman B, Pomeranz B. Basics of acupuncture.Berlin: Springer Science \& Business Media; 2003.

15. Wang GJ, Ayati MH, Zhang WB. Meridian studies in China: a systematic review. J Acupunct Meridian Stud. 2010;3(1):1-9.

16. Beal M. Acupuncture and acupressure Applications to women's reproductive health care. J Nurs Midwifery. 1999;44(3):217-30.

17. Tournaire M, Theau-Yonneau A. Complementary and alternative approaches to pain relief during labor. Evid Based Complement Alternat Med. 2007;4(4):409-17.

18. Lee MK, Chang SB, Kang DH. Effects of SP6 acupressure on labor pain and length of delivery time in women during labor. J Altern Complement Med. 2004;10(6):959-65.

19. Lim CE, Wilkinson JM, Wong WS, Cheng NC. Effect of acupuncture on induction of labor. J Altern Complement Med. 2009;15(11):120914.

20. Gaudet LM, Dyzak R, Aung SK, Smith GN. Effectiveness of acupuncture for the initiation of labour at term: a pilot randomized controlled trial.J Obstet Gynaecol Can. 2008;30(12):1118-23.

21. Azhari S, Abrishami. L., Fadaee. A., Shakeri MT. Efficacy of acupressure on initiating labor in term pregnant women. JMashhad School Nurs Midw. 2009;9(3):207-14.

22. Rabl M, Ahner R, Bitschnau M, Zeisler H, Husslein P. Acupuncture for cervical ripening and induction of labor at term-a randomized controlled trial. Wien Klin Wochenschr. 2001;113(2324):942-6. 